\title{
Application of MCR-ALS to reveal intermediate conformations in the thermally induced $\alpha-\beta$ transition of poly-L-lysine monitored by FT-IR spectroscopy
}

\author{
Mirta R. Alcaráz ${ }^{1,2}$, Andreas Schwaighofer ${ }^{1}$, Héctor Goicoechea ${ }^{2}$, Bernhard Lendl ${ }^{1, *}$
}

${ }^{1}$ Institute of Chemical Technologies and Analytics, Vienna University of Technology, Getreidemarkt 9/164-UPA, 1060 Vienna, Austria.

${ }^{2}$ Laboratorio de Desarrollo Analítico y Quimiometría, FBCB, Universidad Nacional del Litoral-CONICET, Ciudad Universitaria, 3000 Santa Fe, Argentina.

\begin{abstract}
Temperature-induced conformational transitions of poly-L-lysine were monitored with Fourier-transform infrared (FT-IR) spectroscopy between $10^{\circ} \mathrm{C}$ and $70^{\circ} \mathrm{C}$. Chemometric analysis of dynamic IR spectra was performed by multivariate curve analysis-alternating least squares (MCR-ALS) of the amide I' and amide II' spectral region. With this approach, the pure spectral and concentration profiles of the conformational transition were obtained. Beside the initial $\alpha$-helical, the intermediate random coil/extended helices and the final $\beta$ sheet structure, an additional intermediate PLL conformation was identified and attributed to a transient $\beta$-sheet structure.
\end{abstract}

Keywords: Fourier transform infrared spectroscopy, multivariate curve analysis-alternating least squares, secondary structure, conformational analysis, poly-1-lysine

(C) 2019. This is the peer reviewed version of the following article: M.R. Alcaráz, A. Schwaighofer, H. Goicoechea, B. Lendl, Application of MCR-ALS to reveal intermediate conformations in the thermally induced $\alpha-\beta$ transition of poly-1-lysine monitored by FT-IR spectroscopy, Spectrochim. Acta, Part A 185 (2017) 304-309, which has been published in final form at https://doi.org/10.1016/j.saa.2017.05.005. This manuscript version is made available under the CCBY-NC-ND 4.0 license http://creativecommons.org/licenses/by-nc-nd/4.0/ 


\section{Introduction}

Infrared (IR) spectroscopy is a well-established experimental technique for analysis of a wide range of analytes, and provides great sensitivity to small structural changes. However, particularly in condensed phase, this potential often cannot be fully utilized due to the overlap of spectral bands. This is especially the case in protein investigations, because almost all functional groups feature IR active vibrational modes [1,2]. Consequently, different techniques and methods have been devised to decompose or reduce the number of overlapping bands. Experimental techniques to reduce the number of overlapping bands in the IR spectra are difference spectroscopy, where only the bands appear in the spectrum that reflect the difference between two different external conditions, and modulated excitation (ME) spectroscopy [3]. In ME spectroscopy, the evaluation of phase lags of experiments performed at varying excitation frequencies (phase-sensitive detection) allows to identify and verify vibrational components of the underlying dynamic process [4,5]. However, the related experimental effort is complex and tedious. Further, most common methods to decompose broad and overlapping band rely on data processing in order to extract the chemical information from the spectra. Frequently used methods for band separation are second derivative, Fourier self-deconvolution, and curve fitting [6,7]. Particularly for dynamic, timeresolved spectra, two-dimensional correlation spectroscopy (2DCOS) and multivariate curve resolution-alternating least squares (MCR-ALS) have been successfully employed in the last years [8]. 2DCOS is capable of providing resolution enhancement and potentially identifies correlations in the dataset that help with band assignments [2]. However, 2D correlation maps are often unclear and difficult to interpret, thus some art is required to extract significant conclusions from the 2DCOS results [9]. In contrast, MCR-ALS is an iterative soft-modelling method that permits modelling of the dataset [10]. With MCR-ALS, information about multicomponent systems can be obtained by discriminating individual contributions of underlying constituents [11]. An important characteristic of MCR-ALS is that it delivers objective criteria and figures of merit that indicate the success of the modelling [12]. MCRALS further provides sound and meaningful models with chemically interpretable output in the form of spectral profiles of the compounds and the related concentration profiles along the dynamic process, rendering the results easily accessible to the non-chemometric scientific community. During the past years, MCR-ALS has demonstrated to be a powerful chemometric tool for solving problems in data interpretation and analysis. It has been successfully used in combination with various analytical techniques, such as chromatography [13], electrophoresis [14] and flow analysis [15], among others. Particularly in combination with spectroscopic techniques such as IR spectroscopy, MCR-ALS has become a highly valuable technique for the study of dynamic processes due to the possibility to extract pure spectral profiles of the involved components, as well as their varying abundance throughout the process [16-18].

Fourier transform infrared (FT-IR) spectroscopy is a powerful technique for analysis of the structure and dynamics of polypeptides and proteins [19]. The most prominent absorption features of proteins in the IR spectrum are the amide bands, which are caused by vibrations of the peptide group. Among the nine amide bands, the amide I $\left(1600-1700 \mathrm{~cm}^{-1}\right)$ and amide II (1500-1600 $\left.\mathrm{cm}^{-1}\right)$ bands are the most commonly used for secondary structure analysis of proteins and peptides [20]. The differing pattern of hydrogen bonding, dipole-dipole 
interactions and geometric orientations in the $\alpha$-helices, $\beta$-sheets, turns and random coil structures induce different frequencies of the $\mathrm{C}=\mathrm{O}$ and $\mathrm{N}-\mathrm{H}$ vibrations that can be correlated with the respective secondary structural folding [21]. In aqueous solution, the $\mathrm{HOH}$-bending band of water near $1645 \mathrm{~cm}^{-1}$ overlaps with the amide I band in the IR spectrum, consequently measurements of proteins are often performed in $\mathrm{D}_{2} \mathrm{O}$ solution as an alternative. The DODbending band is located near $1200 \mathrm{~cm}^{-1}$, thus creating a region of relatively low solvent absorbance between 1500 and $1800 \mathrm{~cm}^{-1}$. In deuterated solution, the amide II band (predominantly originating from $\mathrm{N}-\mathrm{H}$ vibrations) is shifted from approximately 1550 to $1450 \mathrm{~cm}^{-1}$, then referred to as the amide II' band. Since the amide I band is mainly composed of $\mathrm{C}=\mathrm{O}$ vibrations, the shift is relatively small $\left(5-10 \mathrm{~cm}^{-1}\right)$ compared to the amide II band [22]. In combination with temperature studies, FT-IR has been extensively used to reveal structural changes of proteins and polypeptides induced by increasing temperatures [23-25].

Poly-L-lysine is a widely used model substance for the investigation of secondary structure. It can adopt different conformations depending on a wide range of chemical and physical conditions. Its structural changes induced by external perturbation, e.g., $\mathrm{pH}$ value [26], pressure [27] and temperature [28-30], have been investigated by FT-IR spectroscopy. Further, it has been shown that its conformational state depends on the chain length [29], concentration [30,31] and hydration [3,32] when present in form of a polypeptide film. In aqueous solution at $\mathrm{pH}$ values higher than its $\mathrm{pKa}(10.5)$, the lysine side chain is deprotonated and PLL adopts an $\alpha$-helical conformation at atmospheric pressure. Under these conditions, i.e., $\mathrm{pH}$ values higher than its $\mathrm{pKa}$, upon heating to $50^{\circ} \mathrm{C}$, the structure of PLL transforms into $\beta$-sheets [28,29]. This thermally-induced transition of PLL from $\alpha$-helical to $\beta$-sheet conformation at high $\mathrm{pH}$ values involves an intermediate conformation that has been attributed to extended helices [28,29] or random coil conformation [33]. A further transient $\beta$ sheet component has been previously proposed by ultraviolet resonance Raman (UVRR) spectroscopy [34] as well as by IR spectroscopy after applying a laborious ME-spectroscopy approach, where the conformational transition was stimulated by periodical changes of the water content of an hydrated PLL film followed by phase sensitive detection and 2DCOS [3]. In this work, the temperature-induced conformational change by heating from $10{ }^{\circ} \mathrm{C}$ to $70{ }^{\circ} \mathrm{C}$ of PLL was monitored by using FT-IR spectroscopy. MCR-ALS was employed to distinguish and characterize the involved components in the conformational transition between $\alpha$-helices and $\beta$-sheets. The rigorous application of this chemometric method to IR spectra in the amide I' and amide II' region enabled elucidating an intermediary $\beta$-sheet conformation as a precursor to the final $\beta$-sheet structure. This could be achieved from readily feasible temperature-resolved FT-IR spectra in combination with chemometric analysis by MCR-ALS.

\section{Materials and methods}

\subsection{Reagents and samples}

Poly-L-lysine hydrobromide (PLL, Mw 15000-30000 Da), deuterium oxide ( $\mathrm{D}_{2} \mathrm{O}, 99.9$ atom $\% \mathrm{D}$ ), deuterium chloride solution ( $\mathrm{DCl}, 35 \mathrm{wt} . \%$ in $\mathrm{D}_{2} \mathrm{O} 99$ atom \% D) and sodium deuteroxide (NaOD, 30 wt. \% in $\mathrm{D}_{2} \mathrm{O}, 99$ atom \% D) were purchased from Sigma-Aldrich (Steinheim, Germany). Sodium phosphate tribasic anhydrous $\left(\mathrm{Na}_{3} \mathrm{PO}_{4}\right.$, tech.) was obtained from Alfa-Aesar (Lancashire, UK).

Deuterated phosphate buffer preparation was performed by dissolving an appropriate amount of $\mathrm{Na}_{3} \mathrm{PO}_{4}$ in $\mathrm{D}_{2} \mathrm{O}$, in order to obtain a concentration of $50 \mathrm{mmol} \mathrm{L}^{-1}$, and adjusting the $\mathrm{pH}$ 
with $\mathrm{DCl}$ or $\mathrm{NaOD}$ to 12.0 . PLL solutions were prepared by dissolving $18.0 \mathrm{mg}$ of lyophilized powder directly in $1.0 \mathrm{~mL}$ of $50 \mathrm{mmol} \mathrm{L}^{-1}$ deuterated phosphate buffer $\mathrm{pH} 12.0(\mathrm{pD}=\mathrm{pH}+0.4)$ [35]. If necessary, the $\mathrm{pH}$ of PLL solution was adjusted to 12.0. All solutions were prepared in a glovebox, under constant $\mathrm{N}_{2}$ stream.

pH measurements were carried out by using a pH330i (Wissenschaftlich-Technische Werkstätten $\mathrm{GmbH}$, Weilheim, Germany) potentiometer equipped with a Sentix ${ }^{\circledR}$ Mic-D (Wissenschaftlich-Technische Werkstätten GmbH, Weilheim, Germany) combined glass electrode and temperature probe.

\subsection{Instrumentation and procedure}

The FT-IR measurements were conducted by using a Vertex 80v FT-IR spectrometer (Bruker, Ettllinge, Germany) equipped with a liquid nitrogen cooled MCT-detector, and a $53 \mu \mathrm{m}$ custom-made flow-cell with temperature control. During the measurements, the sample compartment of the spectrometer was flushed with dry air. The recording of spectra was done with a spectral resolution of $2 \mathrm{~cm}^{-1}$ in double-sided, forward-backward, acquisition mode using a Blackman-Harris 3-term apodization function and a zero filling factor of 4 as acquisition parameters. The temperature-controlled experiments were carried out with a custom-made temperature cell consisting of nine thermoelectric cooling elements, stabilized by liquid water. IR single channel spectra of the reference (buffer) and sample (PLL) with a spectral range of $4000 \mathrm{~cm}^{-1}$ and $800 \mathrm{~cm}^{-1-}$ were an average of 450 scans acquired in a temperature range of $10-70{ }^{\circ} \mathrm{C}\left(\Delta \mathrm{T}=2{ }^{\circ} \mathrm{C}\right)$, after an equilibration time of $240 \mathrm{~s}$ at each temperature step. If necessary, water vapour subtraction and baseline correction were performed. For data analysis the software package OPUS 7.2 (Bruker, Ettlingen, Germany) was used.

\subsection{MCR-ALS}

MCR-ALS is a soft-modelling technique that accomplishes the decomposition of multicomponent information of a data matrix by providing a chemically meaningful bilinear model of pure contributions of each component involved in the system [36]. In spectroscopy, the basic assumption of MCR-ALS is the validity of the multicomponent Beer-Lambert law for the studied system [11]. The MCR-ALS analysis can be expressed as a bilinear additive model formed by $\mathbf{c}_{\mathbf{i}} \mathbf{s}_{\mathbf{i}}^{\mathrm{T}}$ dyads, where $\mathbf{s}_{\mathbf{i}}^{\mathrm{T}}$ is the instrumental response of an individual component weighted by the related abundance, $\mathbf{c}_{\mathbf{i}}$. Decomposition is achieved by iterative optimization of the spectroscopic multicomponent data set, expressed as the bilinear model

$$
\mathbf{D}=\mathbf{C S}^{\mathrm{T}}+\mathbf{E}
$$

where $\mathbf{C}$ and $\mathbf{S}$ comprise the concentration and spectral profile, respectively, of all components involved in the system, and $\mathbf{E}$ represents the error or variance left unexplained by the bilinear model [37]. One of the most compelling characteristics of MCR-ALS resolution is its general applicability without prior information about the system under study. However, to achieve chemically meaningful component profiles additional knowledge can be allocated. Decomposition of $\mathbf{D}$ is obtained by iterative optimization of the initial estimates of either $\mathbf{C}$ or $\mathbf{S}$ by using the provided knowledge about the system [38]. This information is introduced through the implementation of chemical or mathematical constraints, such as non-negativity, unimodality, normalization and closure, among others [39]. 
Here, temperature-induced conformational change was monitored by varying the temperature from $10{ }^{\circ} \mathrm{C}$ to $70{ }^{\circ} \mathrm{C}$, every $2{ }^{\circ} \mathrm{C}$. To build the temperature-spectral matrix, IR spectra were restricted to the amide I' and amide II' region $\left(1720-1360 \mathrm{~cm}^{-1}\right)$. In this way, the corresponding matrix for one measurement consisted of $31 \times 750$ data points for the temperature and spectral dimension, respectively. Prior to MCR-ALS modelling, the number of spectrally active components was obtained by applying singular value decomposition (SVD) to the data matrix. Subsequently, to build the initial estimates, an analysis of the purest spectra based on SIMPLISMA methodology was performed [40]. Eventually, to achieve a chemically interpretable solution and contribute to the unique resolution of the components, constraints linked to natural properties of the measurements were applied, i.e., non-negativity in spectral and concentration modes and closure in the concentration mode to account for the mass balance condition.

Data processing and MCR-ALS analysis were performed in MATLAB R2014 (MathWorks,Inc., Natick, MA, 2014). MCR-ALS algorithms were implemented by using MCR-ALS GUI 2.0 graphical interface available at http://www.mcrals.info.

\section{Results and Discussion}

\subsection{IR spectra of temperature-induced conformational change of PLL}

IR spectra of the temperature-induced structural change of PLL were recorded between $10^{\circ} \mathrm{C}$ and $70^{\circ} \mathrm{C}$. In Fig. 1, the IR absorbance spectra in the amide I' and amide II' region are shown. The IR spectrum at $10^{\circ} \mathrm{C}$ shows a band maximum in the amide I' region at $1634.9 \mathrm{~cm}^{-1}$, and band maxima in the amide II' region at $1464 \mathrm{~cm}^{-1}$ and $1442 \mathrm{~cm}^{-1}$, characteristic for the $\alpha$-helical conformation adopted by PLL at these conditions in $\mathrm{D}_{2} \mathrm{O}$-based solution $[3,26,30,41]$. The position of the absorption band for the $\alpha$-helical structure of PLL in the amide I' region is slightly shifted to lower wavenumbers compared to globular proteins (approx. $1652 \mathrm{~cm}^{-1}$ in deuterated solution) [19]. This shift occurs because of the undisrupted intra- and inter-chain coupling due to the high degree of regularity in the helix structure of a

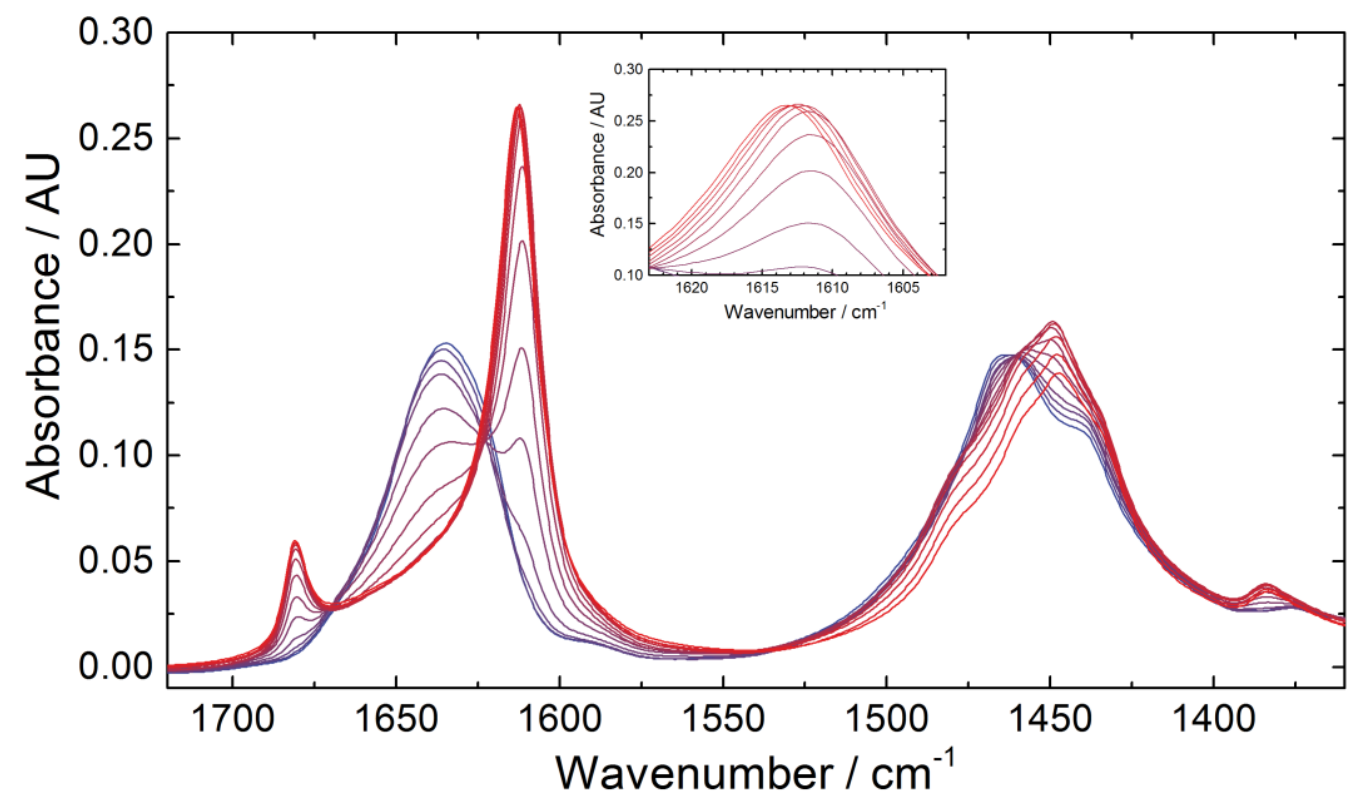

Fig.1. FT-IR spectra of $18 \mathrm{mg} \mathrm{ml}^{-1}$ PLL in deuterated phosphate buffer pD 12.4 recorded as function of the temperature between $10{ }^{\circ} \mathrm{C}$ (blue) and $70{ }^{\circ} \mathrm{C}$ (red). Spectra acquired at 10,18 , 
$26,28,30,32,34,36,40,46,54,62,70^{\circ} \mathrm{C}$ are depicted. The inset illustrates the shift of the low-frequency $\beta$-sheet band to higher wavenumbers in the amide I' region of the IR spectra.

homo-polypeptide [42]. Upon increasing the temperature, the amide I' band shifts to higher wavenumbers which can be correlated with the structural transition from $\alpha$-helix to random coil secondary structure $[33,41]$ or extended helices $[28,29]$.

At higher temperatures, a spectral pattern comprising of a high- and low- frequency band in the amide I' band arises at the expense of the band shapes attributed to the $\alpha$-helical and random coil/extended helix configurations. This feature with band maxima at $1611.5 \mathrm{~cm}^{-1}$ and $1680.8 \mathrm{~cm}^{-1}$ in the amide I' band, accompanied by a band at $1448 \mathrm{~cm}^{-1}$ in the amide II' region, has commonly been attributed to intermolecular antiparallel $\beta$-sheets in PLL [26,27]. The intensity of these bands rises until a solution temperature of $46^{\circ} \mathrm{C}$. At the final temperature range, the band maximum of the low-frequency $\beta$-sheet band shifts to higher wavenumbers (up to $1613.3 \mathrm{~cm}^{-1}$ ) without any further increase of the band height, as illustrated in the inset of Fig. 1. A similar band shift was previously obtained in IR spectra of thermally-induced conformational change of PLL, however it was not further discussed or evaluated [26,29]. For in-depth analysis of the present temperature-resolved IR spectra and further evaluation of the accompanying conformational changes, MCR-ALS analysis was performed.

\subsection{Chemometric analysis by MCR-ALS}

Detailed chemometric analysis of the IR spectra was performed by applying MCR-ALS. This technique has been successfully used for elucidation of intermediate protein folding conformations in dynamic spectroscopic data [43-46]. For the present study, in comparison with other chemometric techniques, MCR-ALS proved to be the most insightful method. This approach offers the possibility to reveal the pure spectral profiles for the compounds involved in the conformational transitions as well as the progression of their relative concentration along the temperature change. Using other chemometric techniques, generally employed for multivariate calibration analysis, such as parallel factor analysis (PARAFAC), loadings with physical interpretation about the system in terms of pure spectral profiles may be obtained for the involved components. However, for the current dataset, this algorithm would not be the best option. A powerful feature of PARAFAC lies in its three-way structure array which is particularly useful in multivariate calibration, but it does not provide any benefit for the analysis of individual matrices. Furthermore, when employing PARAFAC, the constraint referring to the mass balance cannot be applied and the results may not reflect the real situation of the chemical process [47]. On the other hand, algorithms based on partial least square (PLS) are generally suitable for multivariate calibration, and the results as loadings and scores do not carry physically significant information. Then an approximation to the pure constituent spectral or concentration profiles is not feasible [46]. Thus, these algorithms are not applicable when spectral information is desired.

Prior to chemometric analysis, four spectrally active components were identified by SVD. The resulting spectral and concentration profiles obtained by MCR-ALS are presented in Fig. 2. After ALS optimization, the figures of merit obtained ( 0.286 and $99.992 \%$ for lack of fit and percentage of explained variance, respectively) proved the excellent quality of the MCRALS analysis. 
It should be noted that in a former study comprising the MCR-ALS analysis of FT-IR spectra of the temperature-dependent changes of PLL [28], only three components have been identified, most probably because only the amide I region has been considered and spectra acquisition ended at a high temperature of $52{ }^{\circ} \mathrm{C}$. In the present study, both the amide I' and amide II' were included for MCR-ALS analysis, since it was demonstrated that chemometric analysis involving both spectral region provide superior results $[48,49]$.

The amide I' region of the obtained spectral profiles for the four identified components are consistent with $\alpha$-helical $\left(1634.8 \mathrm{~cm}^{-1}\right)$, random coil/extended helices $\left(1636.8 \mathrm{~cm}^{-1}\right)$, transient antiparallel $\beta$-sheet $\left(1611.3\right.$ and $\left.1680.7 \mathrm{~cm}^{-1}\right)$ and antiparallel $\beta$-sheet secondary structure (1613.2 and $1681.0 \mathrm{~cm}^{-1}$ ), respectively. Evaluation of the concentration profiles revealed that the conversion from $\alpha$-helical to random coil/extended helices secondary structure begins immediately after initial heating of the sample. In the same temperature regime, there is a first
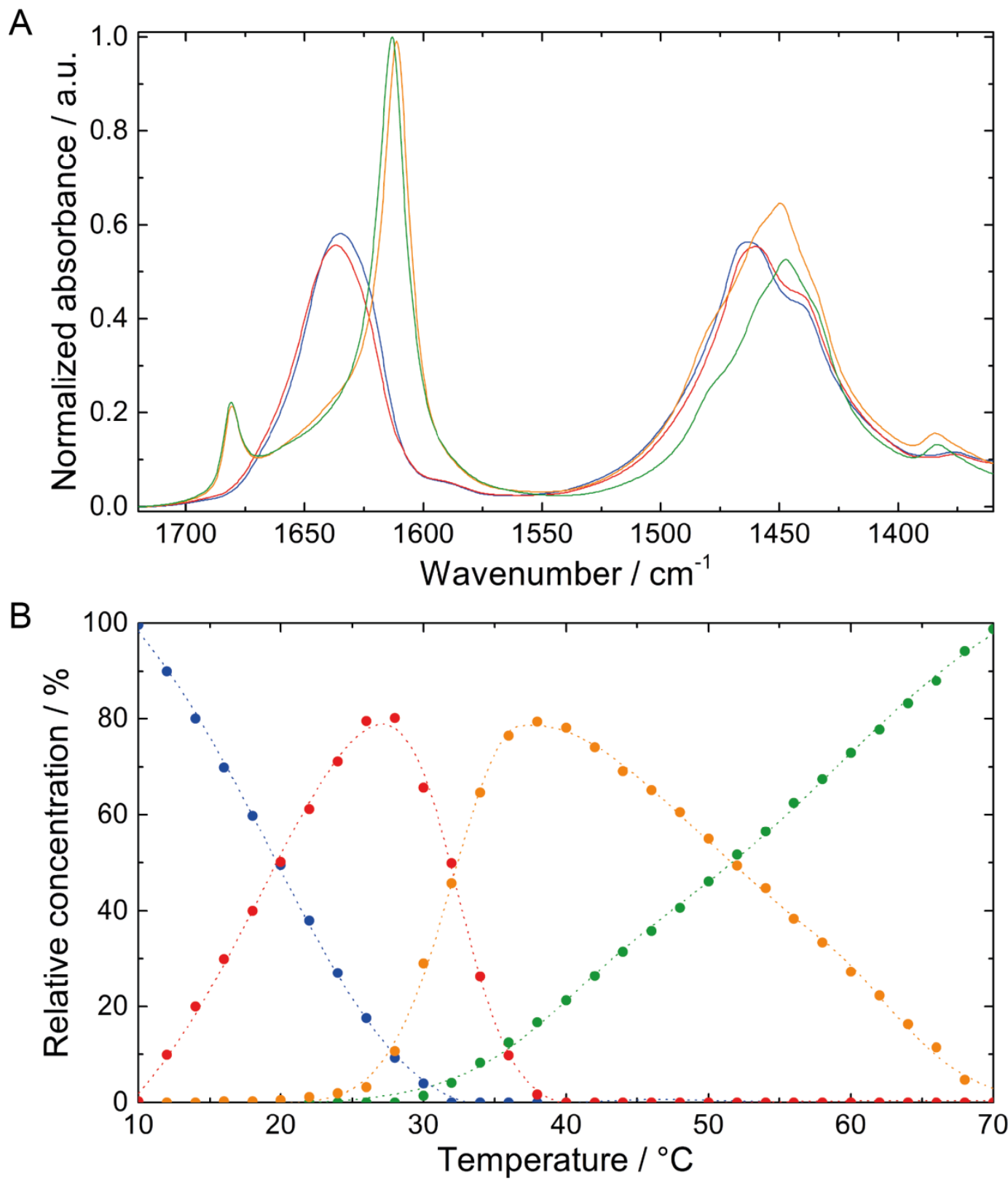
Fig. 2. (A) Spectral and (B) concentration profiles retrieved by MCR-ALS for $18.0 \mathrm{mg} \mathrm{mL}^{-1}$ PLL in deuterated phosphate buffer $\mathrm{pD}$ 12.4. Blue, red, yellow and green lines show individual contributions of $\alpha$-helical, random/extended helices, transient antiparallel $\beta$-sheet and antiparallel $\beta$-sheet conformation, respectively.

indication of a further conformational change to $\beta$-sheet secondary structure in form of the appearance of shoulders at 1611.5 and $1680.8 \mathrm{~cm}^{-1}$ in the amide I' band. When reaching $\sim 32{ }^{\circ} \mathrm{C}$, the entire $\alpha$-helical content is transformed to random/extended helical secondary structure, while the share in $\beta$-sheets continues to increase. This progression coincides with and effectively illustrates the conception that the random/extended helical structure acts as an intermediate in the thermally induced conversion between the initial $\alpha$-helices and the emerging $\beta$-sheets $[26,28,29,34]$. This structural change is also represented in the amide II' region of the spectral profiles. Upon reaching the temperature of $40{ }^{\circ} \mathrm{C}$, the entire protein content is present in form of a transient $\beta$-sheet secondary structure. Finally, between $40^{\circ} \mathrm{C}$ and $70^{\circ} \mathrm{C}$, the transition between this transient $\beta$-sheet component and the final intermolecular $\beta$-sheets takes place. In the FTIR spectra, this conversion is reflected by a band shift without change of absorbance (see inset in Fig. 1). For ensuring that the described band shift originates from the steadily increasing temperature levels in contrast to prolonged exposure to a certain temperature, IR spectra have been recorded at $50{ }^{\circ} \mathrm{C}$ after waiting times of 50 and 100 minutes. Under these conditions, the band shift was not observed (data not shown).

Generally, band shifts of $\beta$-sheet secondary structure have been ascribed to rearrangement of their internal organisation, e.g., from less dense to a more compact $\beta$-sheet structures [50-52]. For PLL, a transient $\beta$-sheet component has previously been identified, when performing MEIR-spectroscopy of an hydrated PLL film upon inducing the conformational change by periodically changing the water content [3]. Further, the present observations concur with temperature-jump/UVRR studies of PLL, where it has been proposed that the temperatureinduced transition of PLL from $\alpha$-helix to $\beta$-sheets not only involves the well-established random/extended helical structure but also a transient $\beta$-strand conformation. With increasing temperature, the water hydrogen bonds of the $\beta$-strands are replaced with interstrand hydrogen bonds, leading to the formation of the final $\beta$-sheet structure $[28,34]$.

\section{Conclusion}

It was proved that MCR-ALS in combination with FT-IR spectroscopy is a powerful technique for monitoring and interpreting protein folding processes. With this chemometric technique, spectral and concentration profiles are obtained by analysis of dynamic IR spectra that can be obtained by a straightforward experimental approach. The potential for deconvolution of overlapping spectral features in IR spectra was demonstrated. A striking benefit is the capability to detect and model intermediate conformations that can otherwise only be isolated by more elaborate experimental approaches or different analytical methods.

Here, the temperature-induced conformational transition of poly-L-lysine was monitored by FT-IR spectroscopy. Spectral and concentration profiles obtained by MCR-ALS identify reasonable IR absorption bands and provide significant insights into the sequence of the 
individual structures upon heating. The obtained results are in accordance with more recent studies addressing the conformational transitions of PLL induced by temperature. 


\section{Acknowledgements}

The authors thank Dieter Baurecht (University of Vienna) for fruitful discussions. Financial support was provided by the Austrian research funding association (FFG) under the scope of the COMET programme within the research project "Industrial Methods for Process Analytical Chemistry - From Measurement Technologies to Information Systems (imPACts)" (contract \#843546). M.R.A. gratefully acknowledges the financial support provided by CONICET.

\section{References}

[1] A. Dominguez-Vidal, M.P. Saenz-Navajas, M.J. Ayora-Canada, B. Lendl, Detection of albumin unfolding preceding proteolysis using Fourier transform infrared spectroscopy and chemometric data analysis, Anal. Chem. 78 (2006) 3257-3264.

[2] A. Barth, C. Zscherp, What vibrations tell about proteins, Q. Rev. Biophys. 35 (2002) $369-430$.

[3] M. Müller, R. Buchet, U.P. Fringeli, 2D-FTIR ATR Spectroscopy of Thermo-Induced Periodic Secondary Structural Changes of Poly-(1)-lysine: A Cross-Correlation Analysis of Phase-Resolved Temperature Modulation Spectra, J. Phys. Chem. 100 (1996) 10810-10825.

[4] D. Baurecht, U.P. Fringeli, Quantitative modulated excitation Fourier transform infrared spectroscopy, Rev. Sci. Instrum. 72 (2001) 3782-3792.

[5] A. Schwaighofer, S. Ferguson-Miller, R.L.C. Naumann, W. Knoll, C. Nowak, PhaseSensitive Detection in Modulation Excitation Spectroscopy Applied to Potential Induced Electron Transfer in Cytochrome c Oxidase, Appl. Spectrosc. 68 (2014) 5-13.

[6] M. Baldassarre, C. Li, N. Eremina, E. Goormaghtigh, A. Barth, Simultaneous Fitting of Absorption Spectra and Their Second Derivatives for an Improved Analysis of Protein Infrared Spectra, Molecules 20 (2015) 12599.

[7] J.T. Pelton, L.R. McLean, Spectroscopic Methods for Analysis of Protein Secondary Structure, Anal. Biochem. 277 (2000) 167-176.

[8] I. Noda, Two-dimensional infrared spectroscopy, J. Am. Chem. Soc. 111 (1989) 81168118.

[9] M.A. Czarnecki, Interpretation of two-dimensional correlation spectra: Science or art?, Appl. Spectrosc. 52 (1998) 1583-1590.

[10] R. Tauler, Multivariate curve resolution applied to second order data, Chemom. Intell. Lab. Syst. 30 (1995) 133-146.

[11] C. Ruckebusch, L. Blanchet, Multivariate curve resolution: A review of advanced and tailored applications and challenges, Anal. Chim. Acta 765 (2013) 28-36.

[12] J. Jaumot, R. Gargallo, A. de Juan, R. Tauler, A graphical user-friendly interface for MCR-ALS: a new tool for multivariate curve resolution in MATLAB, Chemom. Intell. Lab. Syst. 76 (2005) 101-110.

[13] M.R. Alcaráz, G.G. Siano, M.J. Culzoni, A. Munoz de la Pena, H.C. Goicoechea, Modeling four and three-way fast high-performance liquid chromatography with fluorescence detection data for quiatitation of fluoroquinolones in water samples, Anal. Chim. Acta 809 (2014) 37-46. 
[14] M.R. Alcaráz, L. Vera-Candioti, M.J. Culzoni, H.C. Goicoechea, Ultrafast quantitation of six quinolones in water samples by second-order capillary electrophoresis data modeling with multivariate curve resolution-alternating least squares, Anal. Bioanal. Chem. 406 (2014) 2571-2580.

[15] M.R. Alcaráz, A.V. Schenone, M.J. Culzoni, H.C. Goicoechea, Modeling of secondorder spectrophotometric data generated by a $\mathrm{pH}$-gradient flow injection technique for the determination of doxorubicin in human plasma, Microchem. J. 112 (2014) 25-33.

[16] M.R. Alcaráz, A. Schwaighofer, H. Goicoechea, B. Lendl, EC-QCL mid-IR transmission spectroscopy for monitoring dynamic changes of protein secondary structure in aqueous solution on the example of beta-aggregation in alcohol-denaturated alphachymotrypsin, Anal. Bioanal. Chem. 408 (2016) 3933-3941.

[17] J. Diewok, A. De Juan, R. Tauler, B. Lendl, Quantitation of mixtures of diprotic organic acids by FT-IR flow titrations and multivariate curve resolution, Appl. Spectrosc. 56 (2002) 40-50.

[18] J. Kuligowski, G. Quintas, R. Tauler, B. Lendl, M. de la Guardia, Background Correction and Multivariate Curve Resolution of Online Liquid Chromatography with Infrared Spectrometric Detection, Anal. Chem. 83 (2011) 4855-4862.

[19] A. Barth, Infrared spectroscopy of proteins, Biochim. Biophys. Acta, Bioenerg. 1767 (2007) 1073-1101.

[20] K.A. Oberg, J.M. Ruysschaert, E. Goormaghtigh, The optimization of protein secondary structure determination with infrared and circular dichroism spectra, Eur. J. Biochem. 271 (2004) 2937-2948.

[21] S. Bal Ram, Basic Aspects of the Technique and Applications of Infrared Spectroscopy of Peptides and Proteins, in: Infrared Analysis of Peptides and Proteins, American Chemical Society, 1999, pp. 2-37.

[22] H. Fabian, W. Mäntele, Infrared Spectroscopy of Proteins, in: Handbook of Vibrational Spectroscopy, John Wiley \& Sons, Ltd, Hoboken, NJ, USA, 2006.

[23] A. Dong, S.J. Prestrelski, S.D. Allison, J.F. Carpenter, Infrared spectroscopic studies of lyophilization- and temperature-induced protein aggregation, J. Pharm. Sci. 84 (1995) 415 424.

[24] M. Jackson, H.H. Mantsch, Protein-ligand interactions studied by FTIR spectroscopy: Methodological aspects, in: S.E. Harding, B.Z. Chowdhry (Eds.) Protein-ligand interactions, structure and spectroscopy : a practical approach, Oxford University Press, Oxford ;, 2001.

[25] A. Schwaighofer, C. Kotlowski, C. Araman, N. Chu, R. Mastrogiacomo, C. Becker, P. Pelosi, W. Knoll, M. Larisika, C. Nowak, Honey bee odorant-binding protein 14: effects on thermal stability upon odorant binding revealed by FT-IR spectroscopy and CD measurements, Eur. Biophys. J. (2013).

[26] W. Dzwolak, V. Smirnovas, A conformational alpha-helix to beta-sheet transition accompanies racemic self-assembly of polylysine: an FT-IR spectroscopic study, Biophys. Chem. 115 (2005) 49-54.

[27] D. Carrier, H.H. Mantsch, P.T.T. Wong, Pressure-induced reversible changes in secondary structure of poly(L-lysine): An ir spectroscopic study, Biopolymers 29 (1990) 837844.

[28] L. Szyc, S. Pilorz, B. Czarnik-Matusewicz, FTIR-ATR investigations of an alpha-helix to beta-sheet conformational transition in poly(L-lysine), J. Mol. Liq. 141 (2008) 155-159. 
[29] W. Dzwolak, T. Muraki, M. Kato, Y. Taniguchi, Chain-length dependence of alphahelix to beta-sheet transition in polylysine: Model of protein aggregation studied by temperature-tuned FTIR spectroscopy, Biopolymers 73 (2004) 463-469.

[30] A. Schwaighofer, M.R. Alcaraz, C. Araman, H. Goicoechea, B. Lendl, External cavity-quantum cascade laser infrared spectroscopy for secondary structure analysis of proteins at low concentrations, Sci. Rep. 6 (2016) 33556.

[31] T.J. Yu, J.L. Lippert, Peticola.Wl, Laser Raman Studies of Conformational Variations of Poly-L-Lysine, Biopolymers 12 (1973) 2161-2176.

[32] G. Ramer, A. Balbekova, A. Schwaighofer, B. Lendl, Method for Time-Resolved Monitoring of a Solid State Biological Film Using Photothermal Infrared Nanoscopy on the Example of Poly-1-lysine, Anal. Chem. 87 (2015) 4415-4420.

[33] B. Davidson, G.D. Fasman, The Conformational Transitions of Uncharged Poly-Llysine. $\alpha$ Helix-Random Coil- $\beta$ Structure, Biochemistry 6 (1967) 1616-1629.

[34] R.D. JiJi, G. Balakrishnan, Y. Hu, T.G. Spiro, Intermediacy of Poly(l-proline) II and $\beta$-Strand Conformations in Poly(1-lysine) $\beta$-Sheet Formation Probed by TemperatureJump/UV Resonance Raman Spectroscopy, Biochemistry 45 (2006) 34-41.

[35] P.K. Glasoe, F.A. Long, Use of glass electrodes to measure acidities in deuterium oxide, J. Phys. Chem. 64 (1960) 188-190.

[36] S.C. Rutan, A. de Juan, R. Tauler, 2.15 - Introduction to Multivariate Curve Resolution, in: S.D.B.T. Walczak (Ed.) Comprehensive Chemometrics, Elsevier, Oxford, 2009, pp. 249-259.

[37] A. de Juan, S.C. Rutan, R. Tauler, 2.19 - Two-Way Data Analysis: Multivariate Curve Resolution - Iterative Resolution Methods, in: S.D.B.T. Walczak (Ed.) Comprehensive Chemometrics, Elsevier, Oxford, 2009, pp. 325-344.

[38] R. Tauler, M. Maeder, A. de Juan, Multiset Data Analysis: Extended Multivariate Curve Resolution, in: S.D. Brown, R. Tauler, B. Walczak (Eds.) Comprehensive Chemometrics: Chemical and Biochemical Data Analysis, Elsevier, Oxford, 2009, pp. 473505 .

[39] A. De Juan, R. Tauler, Multivariate Curve Resolution (MCR) from 2000: Progress in Concepts and Applications, Crit. Rev. Anal. Chem. 363-4 (2006) 163-176.

[40] W. Windig, J. Guilment, Interactive self-modeling mixture analysis, Anal. Chem. 63 (1991) 1425-1432.

[41] L.P. DeFlores, Z. Ganim, R.A. Nicodemus, A. Tokmakoff, Amide I'-II' 2D IR Spectroscopy Provides Enhanced Protein Secondary Structural Sensitivity, J Am Chem Soc 131 (2009) 3385-3391.

[42] M. Jackson, P.I. Haris, D. Chapman, Conformational Transitions in Poly(L-Lysine) Studies Using Fourier-Transform Infrared-Spectroscopy, Biochim. Biophys. Acta 998 (1989) 75-79.

[43] J. Mendieta, M.S. Diaz-Cruz, M. Esteban, R. Tauler, Multivariate curve resolution: A possible tool in the detection of intermediate structures in protein folding, Biophys. J. 74 (1998) 2876-2888.

[44] J. Mendieta, H. Folque, R. Tauler, Two-phase induction of the nonnative alpha-helical form of beta-lactoglobulin in the presence of trifluoroethanol, Biophys. J. 76 (1999) 451-457.

[45] W. Kessler, R.W. Kessler, Multivariate curve resolution: a method of evaluating the kinetics of biotechnological reactions, Anal. Bioanal. Chem. 384 (2006) 1087-1095. 
[46] A. Borges, R. Tauler, A.d. Juan, Application of multivariate curve resolution to the temperature-induced unfolding of $\alpha$-chymotrypsin, Anal. Chim. Acta 544 (2005) 159-166.

[47] A. Muñoz de la Peña, H.C. Goicoechea, G.M. Escandar, A.C. Olivieri, Fundamentals and Analytical Applications of Multiway Calibration, in: Data Handling in Science and Technology, Elsevier, Amsterdam, 2015, pp. 618.

[48] S. Navea, R. Tauler, E. Goormaghtigh, A. de Juan, Chemometric tools for classification and elucidation of protein secondary structure from infrared and circular dichroism spectroscopic measurements, Proteins: Struct., Funct., Genet. 63 (2006) 527-541.

[49] F. Dousseau, M. Pezolet, Determination of the secondary structure content of proteins in aqueous solutions from their amide I and amide II infrared bands. Comparison between classical and partial least-squares methods, Biochemistry 29 (1990) 8771-8779.

[50] J.C. Ioannou, A.M. Donald, R.H. Tromp, Characterising the secondary structure changes occurring in high density systems of BLG dissolved in aqueous $\mathrm{pH} 3$ buffer, Food Hydrocolloids 46 (2015) 216-225.

[51] R. Sarroukh, E. Goormaghtigh, J.-M. Ruysschaert, V. Raussens, ATR-FTIR: A "rejuvenated" tool to investigate amyloid proteins, Biochim. Biophys. Acta, Biomembr. 1828 (2013) 2328-2338.

[52] L.Z. Polzi, I. Daidone, A. Amadei, A Theoretical Reappraisal of Polylysine in the Investigation of Secondary Structure Sensitivity of Infrared Spectra, J. Phys. Chem. B 116 (2012) 3353-3360. 